\title{
Nutri One-on-One: The Assessment and Evaluation of a Brief One-on-One Nutritional Coaching in Patients Affected by Metabolic Syndrome
}

\author{
Jennifer King, ${ }^{1}$ Jeffrey E. Harris, ${ }^{2}$ David Kuo, ${ }^{3}$ and Farzaneh Daghigh ${ }^{1}$ \\ ${ }^{1}$ Department of Bio-Medical Sciences, Philadelphia College of Osteopathic Medicine, 4170 City Avenue, Philadelphia, PA 19131, USA \\ ${ }^{2}$ West Chester University of PA, 213 Sturzebecker Health Science Center, 855 South New Street, West Chester, PA 19383, USA \\ ${ }^{3}$ Department of Family Medicine, Philadelphia College of Osteopathic Medicine, 4170 City Avenue, Philadelphia, PA 19131, USA
}

Correspondence should be addressed to Farzaneh Daghigh; farzanehd@pcom.edu

Received 31 October 2014; Revised 29 January 2015; Accepted 5 February 2015

Academic Editor: Caryl Nowson

Copyright (C) 2015 Jennifer King et al. This is an open access article distributed under the Creative Commons Attribution License, which permits unrestricted use, distribution, and reproduction in any medium, provided the original work is properly cited.

\begin{abstract}
Nutri One-on-One was a program with the aim to positively modify medical clinic patients' nutritional habits and lifestyles through a brief one-on-one health coaching session. Each session was conducted by utilizing motivational interviewing techniques to allow for tailored nutrition education and goal setting. These sessions were followed by a phone call to participants at 1 month following the session. The outcomes assessed were participant perception of achieving personal nutrition and lifestyle goals, retention of knowledge, and participants' satisfaction with the program. Physicians working in the clinic were assessed for satisfaction with the program. Most of the physicians were generally satisfied with the program and found it to be an asset to their practice. Participants perceived that they achieved their goals, were pleased with the program, and retained knowledge.
\end{abstract}

\section{Introduction}

Obesity is a major health problem across the world, currently affecting two-thirds of U.S. adults [1]. The continual rise in obesity indicates a need for radical change. Currently, there are a variety of factors contributing to the issues of obesity; however, lack of knowledge about nutrition, poor portion control, lack of self-efficacy, and lack of access to nutrition education seem to be the main contributors especially among the low income populations [2]. Therefore, interventions are needed in low socioeconomic, underserved communities.

Evidence shows that providing lifestyle change education and dietary modifications have a notable effect on health outcomes and specifically obesity rates [3]. Primary healthcare settings are ideal locations for addressing the issues of obesity and its comorbidities [4]. Primary care physicians are well situated for effective lifestyle counseling and nutritional education provision. However, a U.S. national survey reveals that "there is a continuing failure to incorporate weight management into clinical medicine practice, especially that of primary care" [4].
Interventions need to be tailored specifically to the individual and nutritional counseling needs to be directed in a way that each participant can adequately address his or her habits, nutritional knowledge, perceived obstacles, self-efficacy, confidence, motivation, and physical concerns in order to be successful [2]. The quality of education and counseling is more important than the quantity or length of the intervention. In fact, evidence shows that even brief meetings with physicians or healthcare professionals show promising lifestyle change effects and "even interventions as short as three minutes can significantly increase change" [3]. However, when addressing duration of interventions it is important that the counseling session is long enough to address client needs, barriers, knowledge deficits, and individual goals [5].

Coach-led one-on-one interventions demonstrate statistically significant effectiveness. This is exemplified by the Ma et al. study where $7 \%$ of the initial target weight loss goal was achieved by $37 \%$ of the one-on-one coach-led intervention participants compared to only $14.4 \%$ of the self-led 
participants [4]. In addition, many studies from USA, Netherlands, Republic of Korea, UK, Germany, and Australia evaluate the continual promising effects of one-on-one intervention on health behavior and the effective elements of brief intervention overall [2-10].

Effective goal setting uses the "SMART framework: mutually establishing behavioral goals that are specific, measurable, achievable, rewarding, and timely" within a one-on-one setting [11]. It is important that the goals determined by the individual are achievable. It is the responsibility of the health coach or healthcare professional to make sure that each goal is connected to outcomes and a reasonable time frame for achievement is established.

Motivational interviewing is a behavior change approach that has been used to promote weight loss and embraces the 5As model. This model is comprised of "five key components: ask, assess, advise, agree, and assist. The model implements a process of counseling that is rooted in the theories of behavior change such as self-management support, readiness assessment, behavior modifications, and self-efficacy enhancement. Motivational interviewing is an evidence-based interviewing method that utilizes patient-driven behavior change to sustain ideal outcomes [11]." The motivational interviewing technique has shown to result in a $1.6 \mathrm{~kg}$ greater weight loss within the first three months of counseling, than in participants who do not receive the motivational interviewing [1]. Therefore, motivational interviewing is an effective method to implement during obesity and nutrition counseling.

Perkins-Porras et al. suggest that behavioral counseling will have different effects or outcomes depending on the baseline stage of readiness in the individual [2]. In order to provide tailored and effective intervention methods specific to the individual, the baseline stage must be determined. The stage of readiness of a person to change dictates which behavior change strategies to use. Motivational interviewing works particularly well for those who are least ready to change.

Glanz et al. state the following: "a comprehensive nutrition intervention in the community requires a multistep approach, including changing social norms and organizational and environmental factors." Therefore, visible and quantifiable change can be expected to come with time [12]. This study of medical clinic patients focused on the effects of one-on-one counseling with a follow-up on achieving their health goals, nutritional knowledge, and overall medical clinic visit satisfaction.

\section{Methods and Materials}

The Nutri One-on-One nutrition education sessions were conducted at four of the Philadelphia College of Osteopathic Medicine (PCOM) healthcare centers, which primarily provide services to the medically underserved populations. The attending physicians at these healthcare centers volunteered to identify and recruit their patients with metabolic syndrome for this study and to fill out a survey at the end of the study. The overall study subjects included adults over the age of eighteen years in healthy mental capacity and exhibiting
TABLE 1: Readiness scores.

\begin{tabular}{lc}
\hline & Score given \\
\hline Not Ready & 1 \\
Moderately Ready & 2 \\
Ready & 3 \\
Currently Making Change & 4 \\
Actively Making Change and Pursuing New Change & 5 \\
\hline
\end{tabular}

one of the five major complications of metabolic syndrome. Subjects were informed of the goals and purpose for the Nutri One-on-One study, asked to give verbal consent to participate, and given the opportunity to withdrawal from the study at any time. This project was approved by the Institutional Review Board of PCOM with ethical considerations.

During nutrition education sessions the health coach gave a brief introduction, explaining the major goals of the study. The subjects were informed of what was expected and of the length of time it would require to complete the session. Participating subjects were also informed of their opportunity to withdraw at any time during the intervention including the one-month follow-up. After the subjects consented, the health coach began to discuss the subject's lifestyle and major health concerns in the one-on-one environment. A personal health and social history was obtained. This information was then used and discussed by both the health coach and the subject to isolate any major health, nutrition, or lifestyle concerns. Then a health form was filled out that included the subject's primary health goal, readiness score, and three health actions. The subjects were encouraged and guided by the health coach to set one primary health goal. This goal was to be relevant to the subject's medical conditions and deemed obtainable by both the subject and the health coach. No specific criteria were set in deeming a health action as "personally obtainable"; however, through discussion with the subject the health coach and subject agreed that the health actions were attainable.

A readiness score value was determined for each subject (Table 1). The stages of change model, which addresses the readiness to change in individuals, was first utilized to treat alcoholism and has recently been applied to dietary behavior. The model consists of "five distinct stages: precontemplation (unaware, not interested in change); contemplation (thinking about change); preparation or decision (making definite plans to change); and action (actively modifying and preventing relapse)" [12]. People vary in their readiness for behavior change in relation to attempting dietary change over time; therefore, methods and steps used to promote healthy changes need to evolve with the individual's progression on the readiness scale.

This score gave a baseline stage of readiness for each participant. The health coach then assessed the health form, subject's health issues, metabolic complications, and primary goal to determine which nutrition education lesson plan to give.

There were ten nutrition education lesson plans available to each subject: Eat Better, Eat the Right Salt, Healthy Portions, Holiday Healthy Eating, Get Active, Lowering 
Cholesterol, Stop Smoking, Eat the Right Carbs, Cooking Class, and Lowering Caloric Intake. The nutrition education lesson plan took approximately fifteen to twenty minutes to deliver and tailor to each individual subject. The nutrition education lesson plan the subject chose was used as a basis for collaboratively determining three health actions associated with his or her primary goal. The health coach then guided the subject by ensuring that the health actions were reasonable, would result in observable positive outcomes, and were perceived as obtainable by the subject.

After completion of the goal setting and health action activities the subject was given a take home flyer relevant to the nutrition education lesson plan received. At the conclusion of the session, the subject was asked to complete a Patient Satisfaction Survey. The survey contained five questions addressing the patient's overall satisfaction with the initial session experience. Participating subjects were asked to report a score, one (strongly disagree) to five (strongly agree), reporting their perception of whether they learned something new, received valuable information, could apply what they learned to achieve a goal, thought the session was long enough to encourage change, and considered the session to be an asset to their doctor's visit.

A follow-up telephone call was conducted approximately one month after the initial session, when the health coach discussed the patient's perceived progress towards his or her overall primary goal, questions, concerns, and obstacles encountered by the subject. The purpose of the one-month follow-up telephone call was to provide continued support and accountability for each subject. The health coach asked the subject to assess how well he or she accomplished each of their three health actions. This was a self-reported score given on a scale of 1 (10\% completion of the health action goal) to 10 (100\% completion of the health action goal) for each action. The health coach then asked the subject if completing his or her health actions and primary goal were still a priority.

Next the health coach instructed the subject to evaluate the value and effectiveness of the Nutri One-on-One Program. The subject gave his or her response to a Likert scale, 1 (not at all valuable) to 5 (extremely valuable). The health coach asked the subject to evaluate his or her overall success in obtaining the three health actions set at the initial session. This self-reported score was also given on a scale, 1 (not at all valuable) to 5 (extremely valuable). The health coach then asked the subject if he or she had taken any other additional actions towards improving his or her health. This yes or no response allowed the health coach to assess how motivated for healthy change the patient was and again answer any questions the subject might have concerning nutrition or further implementation of new goals. At the end of the follow-up the health coach delivered a five-question multiple-choice quiz to the subject. Each of the five questions directly corresponds to one of the five major key messages learned in the nutritional education lesson plan that the subject received during the initial session. The purpose of the educational assessment was to address the key topics learned in the initial session, reinforce the knowledge with the subject, and correct any misconceptions he or she might have. With every question the health coach explained why
TABLE 2: Educational lesson plans.

\begin{tabular}{lc}
\hline Lesson given & Frequency (\%) \\
\hline Eat Better & $2(3 \%)$ \\
Eat the Right Salt & $5(7 \%)$ \\
Healthy Portions & $55(75 \%)$ \\
Holiday Eating & 0 \\
Get Active & $1(1 \%)$ \\
Lower Cholesterol & $7(10 \%)$ \\
Stop Smoking & 0 \\
Reduce Sugar & 0 \\
Cooking Class & $3(3 \%)$ \\
Lower Your Calories & $1(1 \%)$ \\
\hline
\end{tabular}

the answer given by the subject was correct or incorrect, ensuring that the information was thoroughly understood and properly applied to the subject's nutritional habits and knowledge.

At the conclusion of the Nutri One-on-One study, the participating PCOM physicians were given two weeks to fill out an anonymous Physician Satisfaction Survey. The survey addressed the perceptions and attitudes the attending physicians had towards the study, their perceived success of the Nutri One-on-One program, the benefit provided to the patients, and the benefit provided to the healthcare center.

In summary, there were four assessment tools used throughout the Nutri One-on-One study. These were as follows: (1) Patient Satisfaction Survey, (2) educational assessment, (3) subject goal setting and readiness assessment, and (4) Physician Satisfaction Survey. A fifth assessment tool, subjects lab values, was omitted before the start of the study due to inability to schedule clinical follow-ups during this study to obtain anthropometric values after the initial meeting.

\section{Results}

A total of 74 subjects participated in the Nutri One-on-One study, 48 (65\%) were female and $26(35 \%)$ male. The age range was 21 to 79 years of age with an average subject age of 52 years and a standard deviation of \pm 0.13 . A majority of the subjects were hypertensive (77\%) and/or obese (86\%). Diabetes type 2 (53\%) was also prevalent among the population, but only $11 \%$ of the subjects suffered from heart disease. Of the educational lesson plans a majority (75\%) of the subjects chose the "Healthy Portions" lesson plan (Table 2).

Through collecting information for the health form in the personal health and lifestyle history, it was observed that a majority of the participants had good knowledge of healthy foods and how to cook them. Many of the participants (53\%) suffered from Diabetes type 2 and were enrolled in diabetic nutritional classes by their health care insurance companies prior to the study. The number of participants previously enrolled in nutritional classes was not collected, nor was their preexisting nutritional knowledge measured; the nutritional coach purely used this information to further individualize the lesson plan. 
TABLE 3: Patient perceived intervention value.

\begin{tabular}{lc}
\hline & Frequency (\%) \\
\hline Not Valuable & 0 \\
Very Small Value & 0 \\
Somewhat Valuable & $6(12 \%)$ \\
Moderately Valuable & $14(27 \%)$ \\
Extremely Valuable & $31(61 \%)$ \\
\hline Total \# of Subjects & $51(100 \%)$ \\
\hline
\end{tabular}

A readiness score was assigned to each subject at the intervention. Only $9 \%$ of the subject population were not ready to make any nutrition change. The remaining $91 \%$ of the subject population were ready to make varying degrees of nutritional change. The average readiness to change score was a 3, ready to make change (on a scale of 1-5).

The Patient Satisfaction Survey assessed patient satisfaction. $86 \%$ of subjects reported that the information received was very valuable to their overall health, $80 \%$ stated that they could utilize the information received to ensure successful outcomes for their set goals, and $88 \%$ of the subject population reported that the intervention was long enough to create a positive behavioral change.

At the follow-up telephone calls, the percentages of the goals met were determined. At $100 \%$ all of the subjects' set goals would have been achieved and fulfilled. This is a selfreported and self-rated score given at the one-month followup. The participants rated their success for each goal from $0 \%$ (none) to $100 \%$ (all) and an overall average was obtained from all three health goals. On average, subjects met their three health action goals at $63 \% \pm 5 \%$. One subject achieved his goals only to $17 \%$ and another felt that she had achieved her set goals to $97 \%$. The study did not collect objective data on indices to measure their accomplishment of the goals. It solely asked the participant how they perceived their achievement of the goals. A total of 22 subjects were lost after the intervention due to inability to reach them for the followup telephone call.

The subjects related how they felt about goal priority at the follow-up call. 98\% of participants reported that their health goals were still a priority one month after the intervention. Table 3 shows the participants perceived overall value of the intervention at the one-month follow-up session. $100 \%$ of the subject population found the intervention to have some value in facilitating nutrition change.

When asked during the follow-up telephone call if their primary health goal was achieved, $90 \%$ of subjects reported some success in achieving their goals and only $10 \%$ reported not being successful at all with goal attainment. A nutrition education assessment was also administered at the followup telephone call. Each subject was asked five questions corresponding to their specific nutritional education lesson plan. Each of the five questions addressed one of the five major key messages from the lesson, and each question was composed as a multiple-choice question with five possible answer choices. The educational piece assessed the amount of knowledge retained from the lesson plan and provided a percent score that was given for number correct out of 5 . The participant population as a whole was able to correctly recall $75 \%$ of the five key messages for their specific lesson plan received.

Table 4 shows a five-question Likert scale based Physician Satisfaction Survey which was given at the end of the eightmonth long study. All of the physicians $(n=9)$ who participated in the study filled out the Survey. There were 5 physicians who reported that the program had a positive effect on office flow (defined by time allotted for each patient's visit), 3 reported that it had no effect on office flow, and 1 reported the program had a negative effect on office flow. The second question addressed how the attending physicians felt the Nutri One-on-One Program affected the patient's nutrition habits, behavior, and lifestyles. All physicians responded neutrally as they were unable to gain good insight at the time concerning the effects of the program on patients. Eight out of nine (89\%) of the physicians felt that the program was an asset to the services offered at the healthcare centers. $89 \%$ of the physicians stated that the program was extremely valuable to the patients. Lastly, $89 \%$ of the physicians felt the Nutri Oneon-One program had benefits for their patients.

\section{Discussion}

The Nutri One-on-One study focused on knowledge attainment and goal setting to address obesity and its comorbidities [13]. Generally participants were willing to create goals and implement change in their lifestyles to promote positive health lifestyle changes. Toft et al. showed that setting weight loss goals was effective and achievable, which is consistent with the approach of Nutri One-on-One $[14,15]$.

The benefit that the Nutri One-on-One coaching had on the patients was readily apparent throughout the study. During follow-up, subjects commented on notable changes such as weight loss, more energy, and a motivation to keep focusing on achieving personal health goals. They seemed to understand that if they continued, the results would progress further into a greater quality of life, increased longevity, and decreased health expenses. It is important to note that only subjective data on perceived attainment of health goals were obtained.

The readiness score helped focus the health coach on the appropriate method of action for each participant. Ronda et al. have stated that, by defining a readiness score, the overall efficacy and benefit of the program were increased because the health coach addressed change on a level that the subject was willing to implement [16]. In this program 91\% were interested in making varying levels of change; this was most likely due to the fact that they willingly consented to the program. $57 \%$ of participants were interested to learn about the "Healthy Portions" lesson plan during the initial session meeting. This was primarily due to the fact that the majority of subjects were eating their meals at home and had taken multiple nutrition classes giving them a good understanding of which foods were healthy, what foods to avoid, and how to cook in a healthy manner. 
TABle 4: Physician Satisfaction Survey.

\begin{tabular}{lccc}
\hline Questions & $\begin{array}{c}\text { Strongly Disagree, } \\
\text { Negative, } \\
\text { No Change } \\
\text { Not Valuable }\end{array}$ & Neutral & $\begin{array}{c}\text { Strongly Agree, Positively } \\
\text { Large Change, Very } \\
\text { Valuable }\end{array}$ \\
\hline $\begin{array}{l}\text { Clinical value in nutritional coaching and goal setting } \\
\text { Effects of nutritional counseling on office flow }\end{array}$ & 0 & 1 & 8 \\
Observed a noticeable change in patients habits and & 1 & 3 & 5 \\
behavior & 0 & 9 & 0 \\
Nutri One-on-One an asset to services offered & 1 & 0 & 8 \\
Perceived Nutri One-on-One value to patients & 0 & 1 & 8 \\
\hline
\end{tabular}

The 5As model was utilized during this intervention. The intervention proved to be successful for $63 \%$ of the subjects who were motivated by their initial success and results of their health actions so that they personally decided to take additional health actions. Also, $80 \%$ of the participants planned to take additional actions in the future. This was anticipated as Vallis et al. state small success leads to further nutritional motivation in nutritional interventions [11].

Allowing the subjects to set their own health goals in the Nutri One-on-One study ensured that the participant was interested in achieving the goal and taking beneficial actions. The study by Sacerdote et al. established that individual goal setting is effective [8]. During the goal setting, it was important for the health coach to only intervene when the health goal or health actions were unobtainable, not effective, or going to cause harm to the health of the subject. Consistent with Helmink et al., the health coach often recommended keeping goals small during the initial session, so that the goals did not become overwhelming or too extreme for the participant to implement. Many common health goals were seen in the subject population [5]. Among the most prevalent were wanting to lose weight, getting more active, practicing better portion control and meal planning, taking medications more regularly, quitting smoking (a prevalent health goal, yet no participant showed interest in obtaining information on smoking cessation), stopping skipping meals, stopping drinking soda, cooking healthier at home, stopping eating at restaurants, and increasing daily fruits and vegetable intake. The primary health goals and three action plans were all individualized to the participant, therefore, only the overall nutritional education lesson plan data was collected and recorded to give perspective on big picture goals.

The follow-up telephone call was more successful than anticipated, with $80 \%$ of participants successfully contacted. This may be due to the participants' initial willingness to participate in the study, as well as, their preexisting desire for change. This finding coincides with the Glanz et al. study stating that willingness and desire result in change [12].

The attending physicians were all supportive of the project's aim in referring patients and encouraging them to participate in the Nutri One-on-One study, just as Helmink et al. stated that "general practitioners support the notion of these programs" [5]. Nine of the 11 participating attending physicians filled out the physician's survey. Physicians expressed varied perceptions on the success and the effects of the program within the primary healthcare centers; however, specific statements as to why a physician felt positively, negatively, or neutrally toward the Nutri One-on-One program were not discussed. The study indicated benefit to each of the four participating PCOM healthcare centers. Through evaluation of the Patient Satisfaction Survey, patients reported an overall improvement in their primary care visit due to the participation in the Nutri One-on-One study.

There were two key elements presented for the educational assessment portion of the study: the nutritional education lesson plan and the follow-up educational assessment multiple-choice questionnaire. Participants were receptive of the tailored nutritional information; however, the majority of the participants seemed to already have a good idea of what foods were healthy, how to cook in a healthy manner, and how to reduce sodium. Due to $53 \%$ of participants being diagnosed with type 2 Diabetes, a majority of this population had previously participated in diabetes nutrition classes and learned about healthy foods, lowering carbohydrate intake, and avoiding processed foods. Although this knowledge was taught in the class, many did not understand how to apply it to their daily lives specifically, the concepts of portion control, or the importance of meal planning.

There were some limitations of this study such as a large study dropout rate (65\%) during November and December and many subjects expressed that the holidays were too hard to implement change due to stress, travel, and holiday eating. Therefore, it would be wise to address holiday stress and eating in sessions given around the holiday months. In addition, because the study did not look at changes in diet quality in terms of the Healthy Eating Index (HEI), in the future studies parameters such as HEI should be used to examine food habits [17].

A retrospective study would provide valuable insight concerning the impact of the Nutri One-on-One program on its participants. Subjects could be followed up several times up to a year after the initial session about their overall goal achievement, health interest, and nutritional knowledge and be supported through continued motivation for health success. Collecting before and after lab values and anthropometric measurements would give the physicians objective data to evaluate the effectiveness of the program on their patients' nutrition habits and behaviors, long-term results 
of the program and its effects on the metabolic syndrome, and goal maintenance. It would strengthen the study with numerical data not solely reliant on participant self-rated success.

Childhood obesity proved to be very prevalent at the four PCOM healthcare centers. Many families could benefit from family nutritional education. Further work addressing the pediatric population would be necessary and would require parental involvement and action. Therefore, the Nutri Oneon-One program may target an entire family's nutritional habits by extending the duration of this program. Multiple follow-up sessions would offer further motivation and support for an overall long term change. This extension of care and nutritional education would provide continual monitoring of the patient's progress and would have a greater effect on weight loss maintenance for the entire family.

In conclusion, personalized nutritional health coaching through the Nutri One-on-One study has proven to be successful and significant, because an increase in patient primary care visit satisfaction was stated and considerable achievements in self-reported health goals through patient health actions were documented. The program was positively received by both the participating subjects and the physicians, providing evidence that a program such as Nutri One-onOne has a place in the clinical setting that elicits change and is effective for integrative medicine. It also has the potential to have a disseminated effect on the healthcare cost, as $75 \%$ of the US healthcare dollars are currently being spent on diseases caused by obesity. Change needs to begin by employing nutritional education, proper weight loss methods, and maintenance practices that the population can readily use [18].

\section{Conflict of Interests}

The authors declare that there is no conflict of interests regarding the publication of this paper.

\section{References}

[1] D. Laddu, C. Dow, M. Hingle, C. Thomson, and S. Going, "A review of evidence-based strategies to treat obesity in adults," Nutrition in Clinical Practice, vol. 26, no. 5, pp. 512-525, 2011.

[2] L. Perkins-Porras, F. P. Cappuccio, E. Rink, S. Hilton, C. McKay, and A. Steptoe, "Does the effect of behavioral counseling on fruit and vegetable intake vary with stage of readiness to change?" Preventive Medicine, vol. 40, no. 3, pp. 314-320, 2005.

[3] K. Y. Son, C. M. Lee, B. Cho et al., "Effect of aditional brief counselling after periodic health examination on motivation for health behavior change," Journal of Korean Medical Science, vol. 27, no. 11, pp. 1285-1291, 2012.

[4] J. Ma, V. Yank, L. Xiao et al., "Translating the diabetes prevention program lifestyle intervention for weight loss into primary care: a randomized trial," JAMA Internal Medicine, vol. 173, no. 2, pp. 113-121, 2013.

[5] J. H. M. Helmink, J. J. M. Meis, I. de Weerdt, F. N. Visser, N. K. de Vries, and S. P. J. Kremers, "Development and implementation of a lifestyle intervention to promote physical activity and healthy diet in the Dutch general practice setting: the BeweegKuur programme," International Journal of Behavioral Nutrition and Physical Activity, vol. 7, article 49, 2010.

[6] S. J. W. Robroek, F. J. Bredt, and A. Burdorf, "The (cost-) effectiveness of an individually tailored long-term worksite health promotion programme on physical activity and nutrition: design of a pragmatic cluster randomised controlled trial," BMC Public Health, vol. 7, article 259, 2007.

[7] T. P. Wycherley, P. Mohr, M. Noakes, P. M. Clifton, and G. D. Brinkworth, "Self-reported facilitators of, and impediments to maintenance of healthy lifestyle behaviours following a supervised research-based lifestyle intervention programme in patients with type 2 diabetes," Diabetic Medicine, vol. 29, no. 5, pp. 632-639, 2012.

[8] C. Sacerdote, L. Fiorini, R. Rosato, M. Audenino, M. Valpreda, and P. Vineis, "Randomized controlled trial: effect of nutritional counselling in general practice," International Journal of Epidemiology, vol. 35, no. 2, pp. 409-415, 2006.

[9] W. R. Archer, M. C. Batan, L. R. Buchanan et al., "Promising practices for the prevention and control of obesity in the worksite," The American Journal of Health Promotion, vol. 25, no. 3, pp. e12-e26, 2011.

[10] S. C. Bischoff, A. Damms-Machado, C. Betz et al., "Multicenter evaluation of an interdisciplinary 52 -week weight loss program for obesity with regard to body weight, comorbidities and quality of life-a prospective study," International Journal of Obesity, vol. 36, no. 4, pp. 614-624, 2012.

[11] M. Vallis, H. Piccinini-Vallis, A. M. Sharma, and Y. Freedhoff, "Modified 5 As: minimal intervention for obesity counseling in primary care," Canadian Family Physician, vol. 59, no. 1, pp. 2731, 2013.

[12] K. Glanz, R. E. Patterson, A. R. Kristal et al., "Stages of change in adopting healthy diets: fat, fiber, and correlates of nutrient intake," Health Education Quarterly, vol. 21, no. 4, pp. 499-519, 1994.

[13] Centers of Disease Control and Prevention, About BMI for Adults, Centers of Disease Control and Prevention, 2013.

[14] A. L. Marshall, "Challenges and opportunities for promoting physical activity in the workplace," Journal of Science and Medicine in Sport/Sports Medicine Australia, vol. 7, no. 1, pp. 6066, 2004.

[15] U. N. Toft, L. H. Kristoffersen, M. Aadahl, S. L. von Huth, C. Pisinger, and T. Jørgensen, "Diet and exercise intervention in a general population-mediators of participation and adherence: the Inter99 study," The European Journal of Public Health, vol. 17, no. 5, pp. 455-463, 2006.

[16] G. Ronda, P. van Assema, and J. Brug, "Stages of change, psychological factors and awareness of physical activity levels in the Netherlands," Health Promotion International, vol. 16, no. 4, pp. 305-314, 2001.

[17] P. M. Guenther, S. I. Kirkpatrick, J. Reedy et al., "The healthy eating Index-2010 is a valid and reliable measure of diet quality according to the 2010 dietary guidelines for Americans," The Journal of Nutrition, vol. 144, no. 3, pp. 399-407, 2014.

[18] J. M. Olsen and B. J. Nesbitt, "Health coaching to improve healthy lifestyle behaviors: an integrative review," American Journal of Health Promotion, vol. 25, no. 1, pp. el-e12, 2010. 

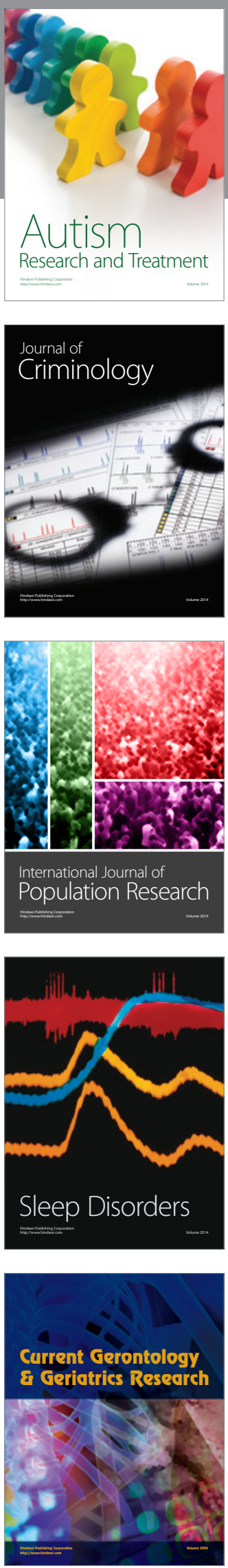
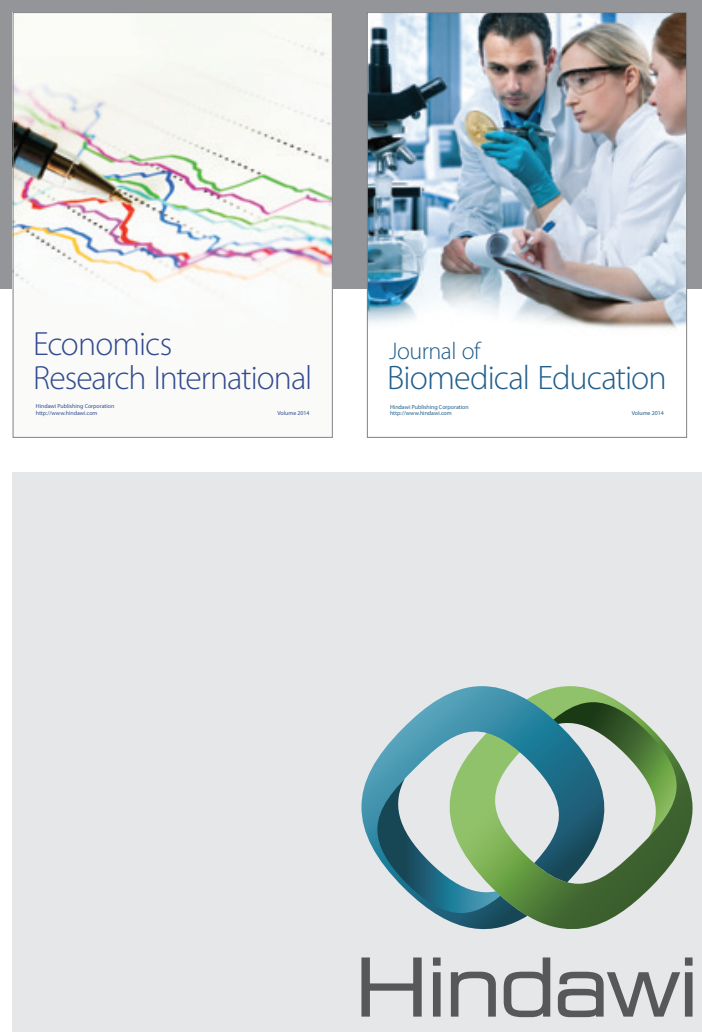

Submit your manuscripts at

http://www.hindawi.com
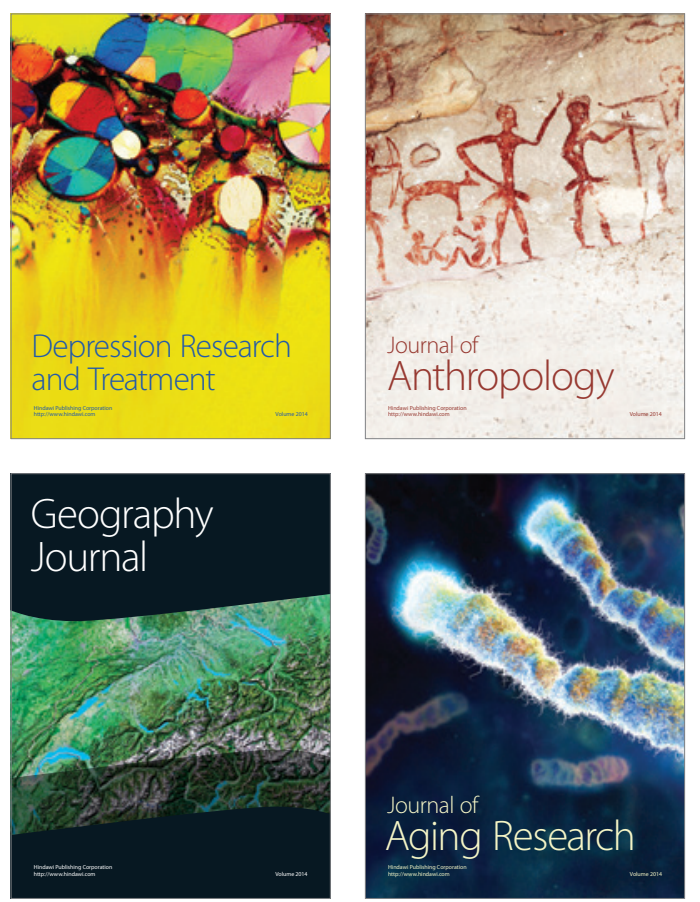
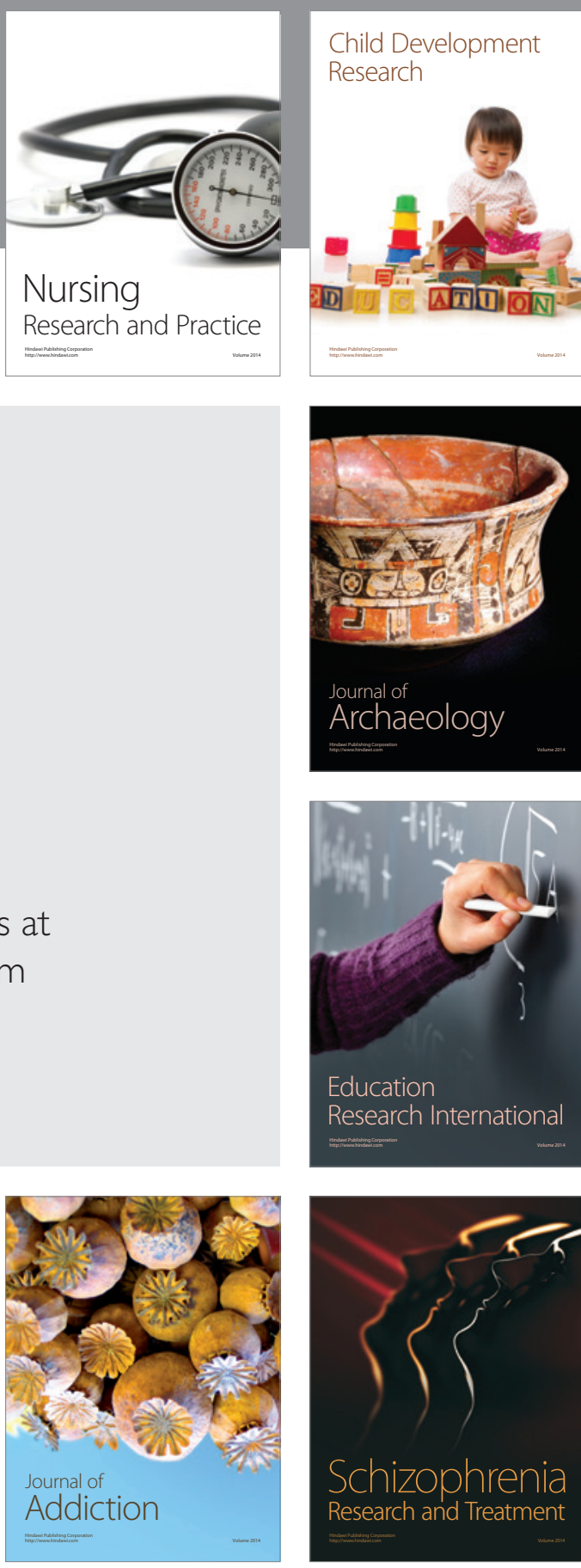

(D)
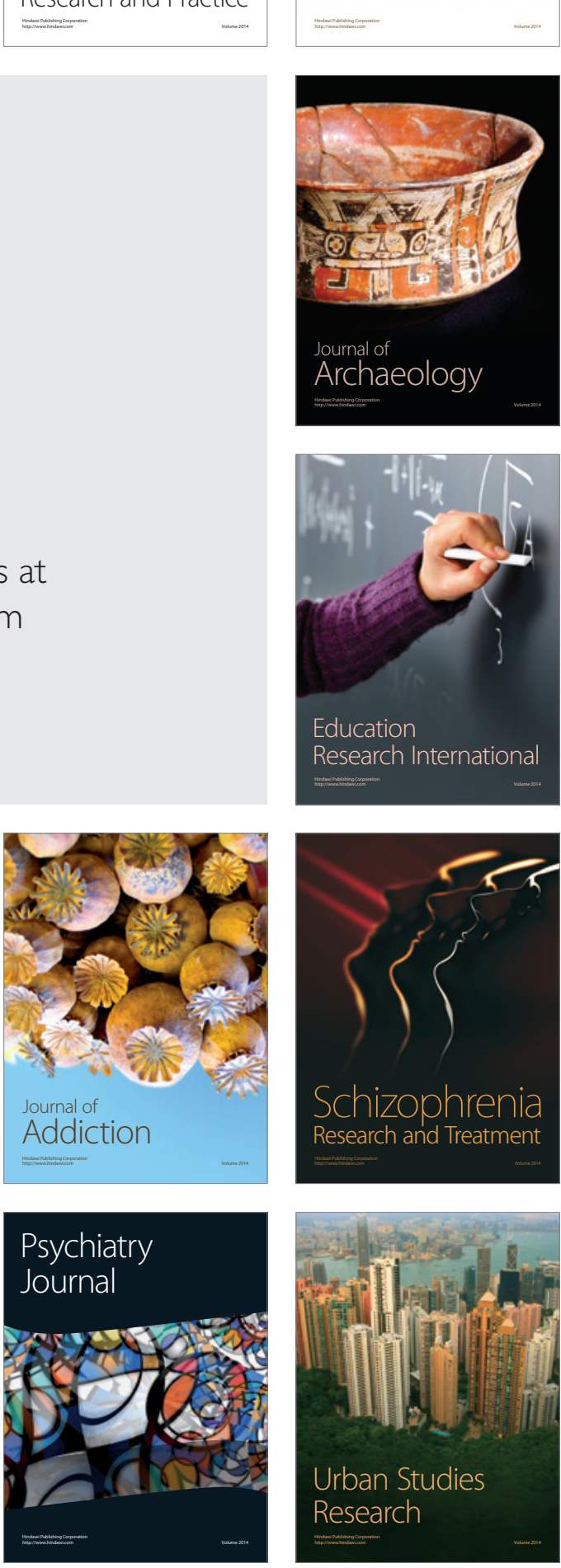